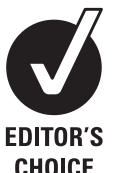

CHOICE
${ }^{1}$ Division of Preventive and Behavioral Medicine, University of Massachusetts Medical School, Worcester, Massachusetts, USA ${ }^{2}$ Division of Pulmonary, Allergy and Critical Care Medicine, University of Massachusetts Medical School, Worcester, Massachusetts, USA ${ }^{3}$ Department of Quantitative Health Sciences, University of Massachusetts Medical School, Worcester, Massachusetts, USA

\section{Correspondence to} Dr James Carmody, University of Massachusetts Medical School, 55 Lake Avenue North, Worcester, MA 01655, USA james.carmody@umassmed.edu

Received 25 March 2011 Accepted 28 March 2012 Published Online First 27 April 2012

\title{
Effect of mindfulness training on asthma quality of life and lung function: a randomised controlled trial
}

\author{
Lori Pbert, ${ }^{1}$ J Mark Madison, ${ }^{2}$ Susan Druker, ${ }^{1}$ Nicholas Olendzki, ${ }^{1}$ Robert Magner, \\ George Reed, ${ }^{1}$ Jeroan Allison, ${ }^{3}$ James Carmody ${ }^{1}$
}

\section{ABSTRACT \\ Background This study evaluated the efficacy} of a mindfulness training programme (mindfulnessbased stress reduction (MBSR)) in improving asthmarelated quality of life and lung function in patients with asthma.

Methods A randomised controlled trial compared an 8-week MBSR group-based programme $(n=42)$ with an educational control programme $(n=41)$ in adults with mild, moderate or severe persistent asthma recruited at a university hospital outpatient primary care and pulmonary care clinic. Primary outcomes were quality of life (Asthma Quality of Life Questionnaire) and lung function (change from baseline in 2-week average morning peak expiratory flow (PEF)). Secondary outcomes were asthma control assessed by 2007 National Institutes of Health/National Heart Lung and Blood Institute guidelines, and stress (Perceived Stress Scale (PSS)). Follow-up assessments were conducted at 10 weeks, 6 and 12 months.

Results At 12 months MBSR resulted in clinically significant improvements from baseline in quality of life (differential change in Asthma Quality of Life Questionnaire score for MBSR vs control: 0.66 (95\% $\mathrm{Cl} 0.30$ to $1.03 ; \mathrm{p}<0.001)$ ) but not in lung function (morning PEF, PEF variability and forced expiratory volume in $1 \mathrm{~s}$ ). MBSR also resulted in clinically significant improvements in perceived stress (differential change in PSS score for MBSR vs control: $-4.5(95 \% \mathrm{Cl}-7.1$ to $-1.9 ; p=0.001))$. There was no significant difference $(p=0.301)$ in percentage of patients in MBSR with well controlled asthma $17.3 \%$ at baseline to $19.4 \%$ ) compared with the control condition $17.5 \%$ at baseline to $7.9 \%$ ).

Conclusions MBSR produced lasting and clinically significant improvements in asthma-related quality of life and stress in patients with persistent asthma, without improvements in lung function.

Clinical Trial Registration Number Asthma and Mindfulness-Based Reduction (MBSR) Identifier: NCT00682669. clinicaltrials.gov.

\section{INTRODUCTION}

Asthma places considerable demands on patients, and interventions to facilitate adjustment to the disease may be important in asthma management even when lung function does not improve. ${ }^{1}$ Psychosocial factors are also implicated. The elevated perceived stress prevalent in patients with asthma negatively affects their quality of life (QOL) and is strongly associated with reduced

\section{Key messages}

What is the key question?

- Can a mind-body training programme bring about a lasting improvement in quality of life and lung function in patients with asthma?

What is the bottom line?

- Compared with an educational control, a mind-body training programme was found to result in lasting improvements in patients' quality of life.

Why read on?

- This is the first report of a controlled trial of a mind-body training programme that used an active control programme, followed patients for 12 months, and included clinically accepted disease outcome markers. It has significant implications for the clinical management of asthma.

medication adherence and worse asthma control, ${ }^{2}$ and with over-perceiving dyspnoea and respiratory symptoms unaccompanied by objective measures. ${ }^{3}$ To limit exacerbations related to these factors, patients need to be able to discriminate between their asthma symptoms and the associated affectrelated sensations and cognitions ${ }^{4}$; nevertheless, self-management programmes typically focus on education about external triggers and medication usage. While cognitive behavioural therapy appears to have a positive effect on asthma QOL and relaxation therapy reduced 'as needed' medication use, the heterogeneity and low quality of studies of the complementary approaches used by $40 \%$ of patients with asthma preclude firm conclusions on their role in asthma. ${ }^{5} 6$

Mindfulness training involves learning to recognise and discriminate between components of experience, including thoughts, feelings, and sensations, and developing a non-reactive awareness of these. Mindfulness-based stress reduction (MBSR) is a widely available group-based mindfulness training programme that reduces perceived stress, disease-related distress and reported medical symptoms in a range of chronic diseases, ${ }^{7}$ but has not been studied for its effect on asthma QOL and management. We hypothesised that MBSR would result in greater $\mathrm{QOL}$ and lung function improvements compared with an education control condition. 


\section{METHODS}

\section{Study sample}

Participants were adult patients recruited between October 2006 and December 2007 from primary and pulmonary care clinics at UMass Memorial Health Care (UMMHC) in Worcester, Massachusetts, USA.

Inclusion criteria were physician-documented asthma with an objective indicator of bronchial hyper-responsiveness (positive methacholine challenge test, $\geq 12 \%$ improvement in forced expiratory volume in $1 \mathrm{~s}\left(\mathrm{FEV}_{1}\right)$ or forced vital capacity $(\mathrm{FVC})$ in response to bronchodilator, or $20 \%$ variability in diurnal peak expiratory flow (PEF) variation), or $\geq 12 \%$ improvement in $\mathrm{FEV}_{1}$ in response to inhaled bronchodilator on spirometry at study entry; and met 2007 National Institutes of Health/National Heart Lung and Blood Institute (NIH/NHLBI) ${ }^{8}$ criteria for mild, moderate, or severe persistent asthma.

Patients were excluded if they had intermittent asthma (symptoms less than once/week, brief exacerbations, nocturnal symptoms $\leq$ twice/month, and normal lung function between episodes); smoked in the past year; other lung diseases; current treatment for symptomatic cardiovascular disease; history of a positive tuberculosis test; participated in MBSR and/or practicing meditation regularly.

Figure 1 shows the flow of participants. Eighty-three proceeded to randomisation. Follow-up occurred between April 2007 and February 2008. The University of Massachusetts Medical School Institutional Review Board approved the protocol. Participants gave informed consent and continued to be managed by their physician.

\section{Intervention groups}

The intervention was MBSR; the control was a Healthy Living Course. Group assignment was by a random allocation scheme with block sizes of four and six.

MBSR consisted of 8 weekly $2.5 \mathrm{~h}$ sessions, and an all-day (6 h) session in week 6. ${ }^{9}$ To enhance generalisability, participants were integrated into regularly scheduled UMMHC MBSR classes and had approximately two study and 28 non-study
Figure 1 Consort diagram. HLC, healthy living course; MBSR, mindfulness-based stress reduction.

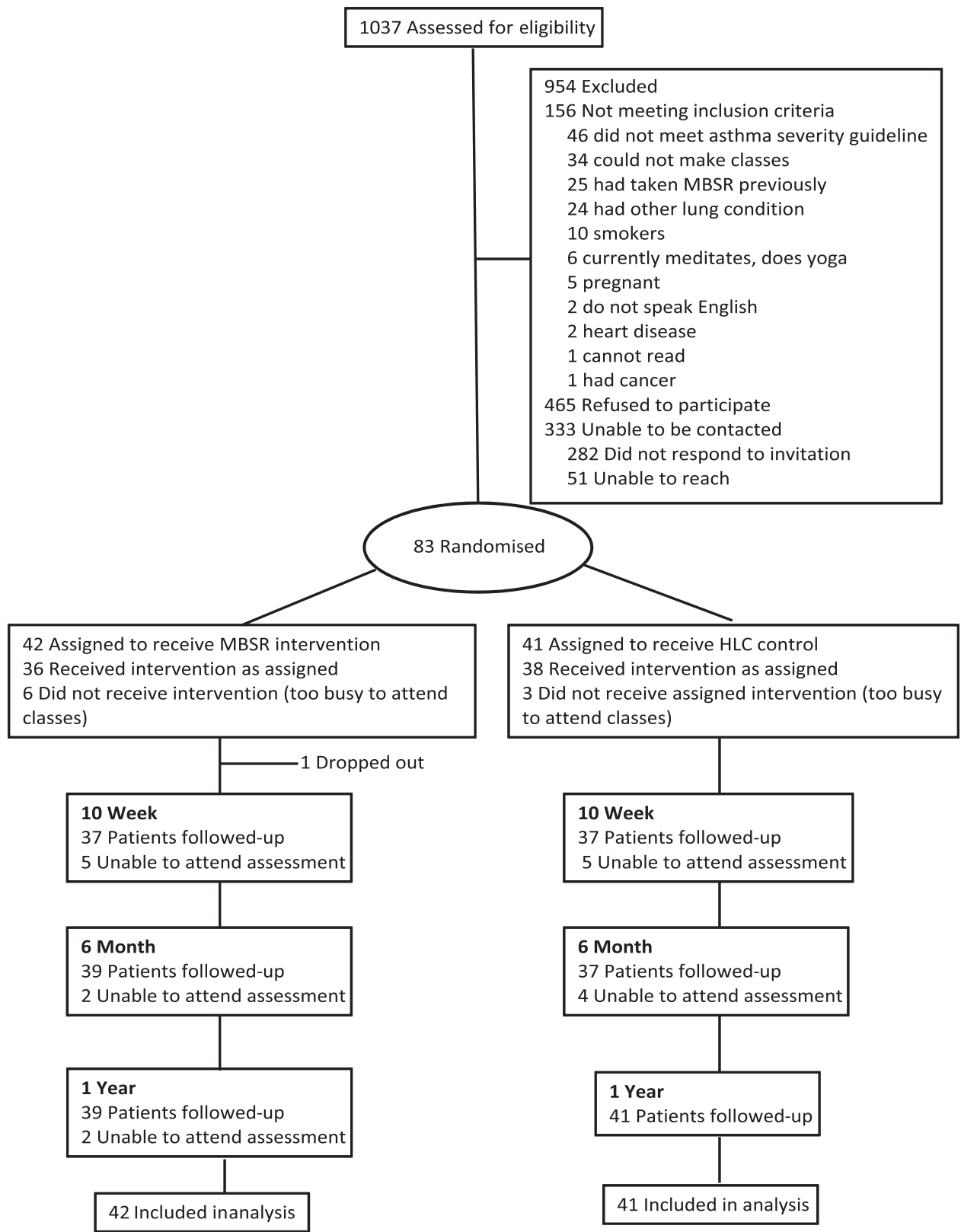


participants. Mindfulness training was through the following techniques: body scan, when attention is systematically moved through the body to bring awareness to sensations; sitting meditation focusing on awareness of breathing, thoughts and feelings; and gentle stretching exercises to develop awareness during movement. The classes emphasised integration of mindfulness into everyday life to support coping with symptoms and stress. Two CDs containing guided mindfulness exercises were provided to be practiced for $30 \mathrm{~min}, 6$ days/week.

To control for as many non-specific factors as possible, we developed a healthy living course (HLC) matched for time, instructor attention and format. Classes consisted of lectures and discussion of self-care topics: healthy nutrition; physical activity; coping with stress (not including mindfulness); sleep hygiene; balancing work and personal life; and living a drug-free life. Homework was assigned consistent in time with MBSR. To match the MBSR mix of participants, HLC was offered to community members in addition to study participants and consisted of approximately 7 study and 18 non-study participants. Classes were held during the same timeframe as MBSR to control for seasonal variation.

\section{Outcome measures}

Assessments occurred at baseline, and at 10 weeks and 6 and 12 months post baseline by evaluators blind to treatment assignment.

Primary outcomes were QOL and lung function. The Asthma Quality of Life Questionnaire (AOOL) ${ }^{10}{ }^{11}$ (32-items) assesses the degree to which important activities were limited by asthma during the last 2 weeks in four domains: activity limitations, asthma symptoms, emotional function and environmental exposure. The overall QOL score, an average of the domains, is valid, reliable and sensitive to changes in asthma symptoms. ${ }^{10}$ The minimum important change in overall QOL score and each of the domains is $0.5^{11}$

Lung function was assessed by change from baseline in 2-week average morning PEF (litres/min), consistent with studies by the National Heart, Lung, and Blood Institute's Asthma Clinical Research Network (ClinicalTrials.gov). While correlation with $\mathrm{FEV}_{1}$ is not always ideal, PEF is easily performed at home and often used as an indicator of lung function. Additional lung function measures were spirometry and PEF variability. Spirometry was performed in the UMMHC Pulmonary Diagnostic Laboratory according to American Thoracic Society guidelines $^{12}$ and assessed $\mathrm{FEV}_{1}$ and FVC. PEF variability compared the minimum morning pre-bronchodilator PEF with the recent best (day's highest minus lowest/mean), providing an amplitude per cent mean. ${ }^{13}$

Secondary outcomes were perceived stress, and asthma control classification based on $2007 \mathrm{NIH} / \mathrm{NHLBI}$ criteria. ${ }^{8}$ Those in a less well controlled category for any component were classified at the higher category. The 10-item Perceived Stress Scale (PSS) ${ }^{14}$ assessed the degree to which the person appraised their coping resources as insufficient to meet the demands of events in the past month.

At each assessment, participants recorded frequency of asthma rescue medication use (short-acting bronchodilators) over a 14-day period, and days of work or school missed due to asthma. Asthma exacerbations were assessed by self-reported initiation of prednisone in the last 30 days.

\section{Analysis}

Analyses were carried out using Stata V.10.1 (Stata Corporation). This was a pilot study powered at the $10 \%$ significance level for a mean difference in AQOL between groups of $<0.5,{ }^{11}$ and a difference in mean per cent change in PEF of $10 \% .{ }^{15}$ At the $10 \%$ significance level, there was $80 \%$ power with $n=35 /$ group. At the $5 \%$ significance level the effective power was $70 \%$. With a predicted $15 \%$ dropout, the recruitment goal was 42 /group. A significance threshold of $\mathrm{p}<0.05$ was used for all analyses. Baseline distributions of important patient characteristics were compared by study group based on non-missing data. T-tests compared AQOL, $\mathrm{FEV}_{1}$, per cent predicted $\mathrm{FEV}_{1}$ and perceived stress, and Wilcoxon rank sum tests compared variables with skewed distributions (short short-term inhaler use, days absent due to asthma). Fisher's exact tests compared all categorical variables.

Prednisone use differed between groups at baseline and was included in final models if associated with time trends and altered estimates of study arm effects.

For the peak flow/medication form and spirometry, there were up to 21 missing data points at follow-up. For short-term rescue medication use, 2-week average morning PEF, PEF variability, and $\mathrm{FEV}_{1}$, missing values were extrapolated using the slope of the two closest non-missing values; for 10 patients, single nonmissing values were carried forward to all subsequent time points. The results presented are from these imputed models. Difference in improvement in AQOL (by 1 or 1.5 points) at 12 months was tested using Fisher's exact test. Class attendance was compared using the Wilcoxon rank-sum test.

The basic analytic strategy was to compare differential trends over time for the intervention versus comparison group. The main outcomes were derived from a difference-of-difference analysis representing over-time change in the intervention group after accounting for simultaneous change in the comparison group. Trends over time in AOOL, PSS, PEF variability, morning $\mathrm{PEF}$ and $\mathrm{FEV}_{1}$ were analysed using linear mixed models with random intercepts and slopes, with participant as the random effect. Each outcome was modelled as a function of group, categorical time and the group-time interaction. The grouptime coefficients reflected differential over-time change for the intervention versus comparison group. Time coefficients reflected over-time change in the comparison group, while the sum of time and group-time coefficients provided change in the intervention group. Dichotomous outcomes (well controlled asthma, prednisone use in past 30 days and long-term controller use) were analysed using Generalised Estimating Equation logistic regression. Times/week using short-term rescue medication was modelled with Generalised Estimating Equation negative binomial regression. For all models, clustering was on participant and an exchangeable correlation structure was assumed. Each outcome was modelled as a function of group, categorical time, and the group-time interaction. The model for rescue medication also included baseline prednisone use and the interaction of baseline prednisone use and time as covariates. Estimates of ORs, incidence rate ratios and CIs were derived from the models.

\section{RESULTS}

\section{Baseline characteristics}

The 56 women and 27 men had a mean age of 52.8 years (see table 1). The majority were white $(93.8 \%)$, non-Latino $(92.3 \%)$ and had either some college or a college or advanced degree (68.8\%). Per cent with well controlled asthma was very low $(7.4 \%)$, consistent with the exclusion of intermittent or well controlled asthma. Of those whose asthma was not well controlled, $80.0 \%$ in HLC and $83.8 \%$ in MBSR were on inhaled corticosteroid or oral prednisone. The number of years taking 
Table 1 Baseline socio-demographical and clinical characteristics

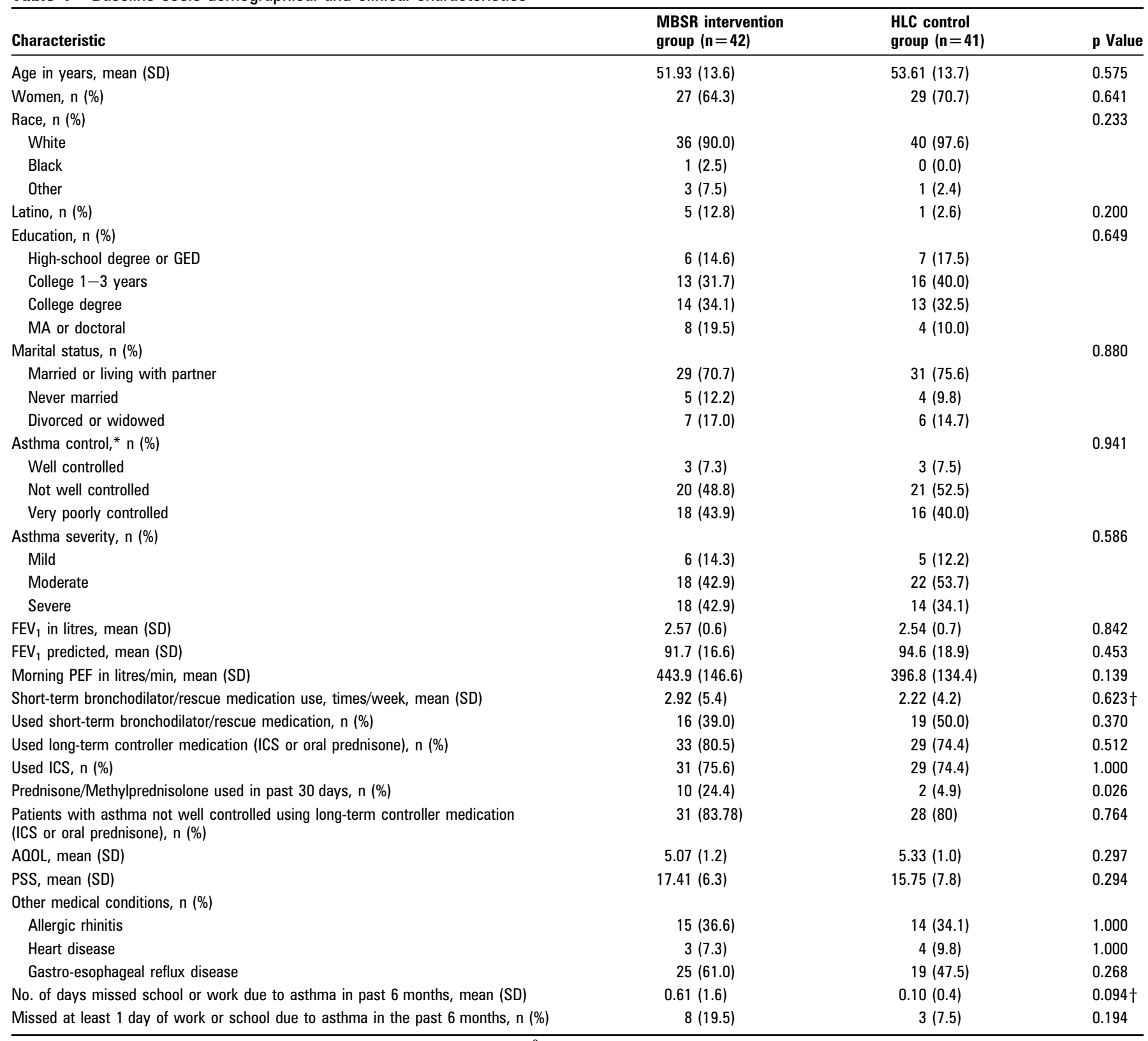

${ }^{*}$ Asthma control was assessed based on the National Heart Lung and Blood Institute ${ }^{8}$ criteria. Presented are per cent classified as well controlled (vs not well controlled or very poorly controlled)

†Rank-sum test.

AOOL, Asthma Quality of Life; FEV 1 , forced expiratory volume in 1 s; GED, general education diploma; HLC, healthy living course; ICS, inhaled corticosteroid; MA, Master of Arts degree; MBSR, mindfulness-based stress reduction; PEF, peak expiratory flow; PSS, Perceived Stress Scale.

asthma medication was 13.3. Mean baseline AOOL (5.20 overall; 5.46 men, 5.08 women) was similar to a cross-sectional study of US adults with asthma. ${ }^{16}$ Mean perceived stress (16.6) was 0.5 SD above the US norm. ${ }^{17}$ The only significant difference between arms in demographical and clinical characteristics was prednisone/Methylprednisolone use in the past 30 days (MBSR $24.4 \%$ vs HLC $4.9 \%, p=0.026$ ). The two conditions did not differ on any other asthma control measures.

\section{Primary outcomes}

Asthma-related $\mathrm{QOL}$

At 12-month follow-up, overall AOOL had significantly improved in the MBSR group compared with controls, increasing by 0.72 and 0.06 respectively (differential change of $0.66, \mathrm{p}<0.001$ ) (see table 2 and figure 2 ). The MBSR group also improved in three of the four AQOL components: activity limitations (differential change of $0.66, p=0.0 .003$ ), symptoms (differential change of $0.63, \mathrm{p}=0.002$ ) and emotional function (differential change of $0.81, p=0.002$ ) (see table 2). At 12 months the effect of MBSR versus control on overall AQOL (differential change of 0.66 ) was $>0.50$, which is considered a clinically significant improvement, and more patients in the MBSR group achieved moderate ( $\geq 1$ point) ( $34 \%$ vs $12 \%, p=0.031$ ) and large improvements in AQOL ( $\geq 1.5$ points) $(18 \%$ vs $5 \%, p=0.080) .{ }^{11}$

\section{Lung function}

The primary lung function measure (2-week average morning $\mathrm{PEF}$ (litre/min)) did not improve in the MBSR group compared with controls; changes from baseline were -4.2 and 6.5 respectively (differential change at 12 months $-10.7, p=0.588$ ) 
Table 2 Asthma Quality of Life (AOOL): model-derived estimates for baseline, follow-up and change from baseline

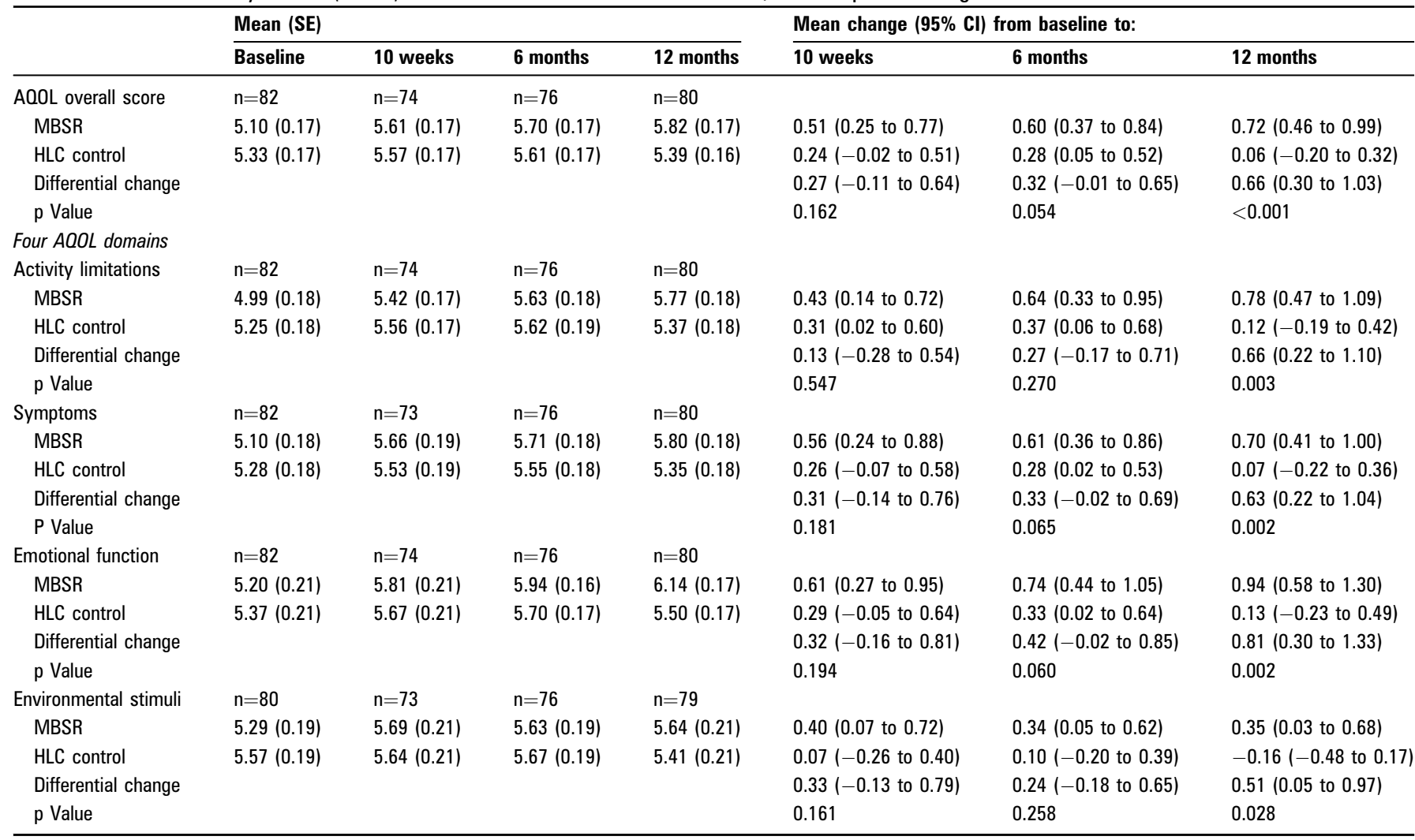

Differential change was calculated as the difference in change from baseline for the intervention versus change from baseline for the comparison group. The differing numbers of individuals included in each outcome analysis is due to the A0OL allowing for differing numbers of missing responses for each subscale.

HLC, healthy living course; MBSR, mindfulness-based stress reduction.

(see table 3). Similarly, effects were not demonstrated in PEF variability (amplitude \% mean) or $\mathrm{FEV}_{1}$ (litres), with respective changes of $-0.27 \%$ in the MBSR group and $-0.05 \%$ in the control group (differential change of $-0.22 \%, p=0.879$ ), and 0.04 in the MBSR group and 0.02 in the control group

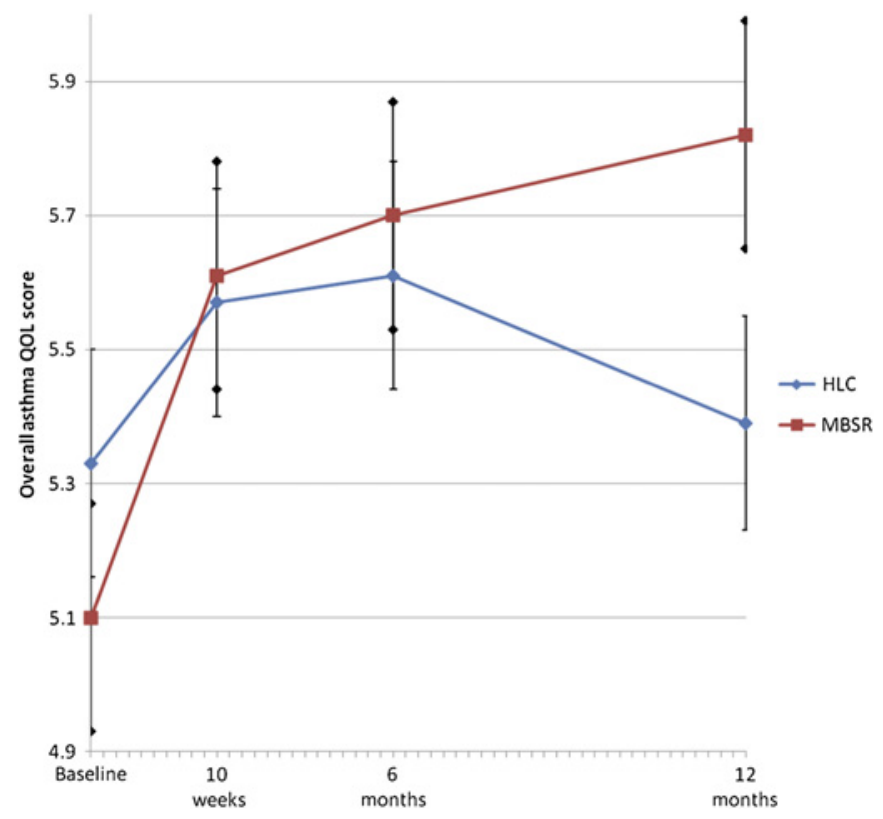

Figure 2 Overall asthma quality of life (AOOL). HLC, healthy living course; MBSR, mindfulness-based stress reduction. (differential change of $0.01, \mathrm{p}=0.809$ ) (see table 3 ). Adherence to PEF monitoring, defined as completing at least one full day of monitoring, was $78 \%$ at 10 weeks, $77 \%$ at 6 months and $78 \%$ at 12 months.

\section{Secondary outcomes}

Asthma control

The percentage of well controlled asthma in the MBSR group went from $7.3 \%$ at baseline to $19.4 \%$ at 12 -month follow-up compared with $7.5 \%$ to $7.9 \%$ in the controls. This differential change was not significant ( $p=0.301)$ (see table 4 and figure 3 ).

The percentage of patients in the MBSR group using longterm controller medication decreased from $80.5 \%$ at baseline to $71.4 \%$ at 12 -month follow-up, while the percentage of controls increased from $74.4 \%$ to $81.25 \%$. This differential change was not significant $(\mathrm{p}=0.064)$ (data not shown).

\section{Perceived stress}

At 12-month follow-up, perceived stress (PSS) had significantly improved in the MBSR group compared with the controls, decreasing by 4.3 and increasing by 0.2 respectively (differential change of $-4.5, p=0.001$ ) (see Table 4 and figure 4).

Asthma medications, exacerbations, days of work/school missed In an unadjusted model, there were no significant differences in change between conditions in use of rescue medications (bronchodilators) ( $p=0.095)$ or prednisone for asthma exacerbations $(p=0.084)$ (see table 4). However, when adjustment was made for prednisone use in the 30 days before baseline assessment, the MBSR group showed a significant decrease compared with the control group in use of rescue medications at 12 months 
Table 3 Lung function: model estimates for baseline, follow-up and change from baseline

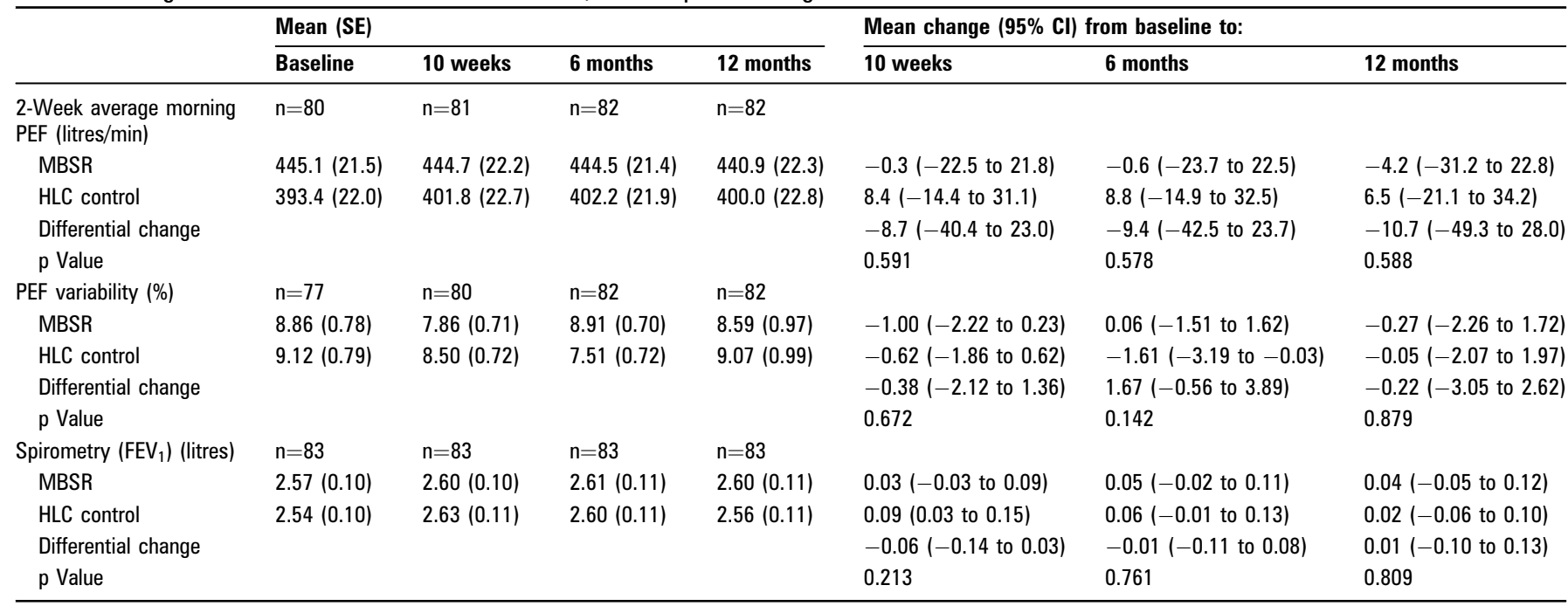

Differential change was calculated as the difference in change from baseline for the intervention versus change from baseline for the comparison group. The differing numbers of individuals included in each outcome analysis is due to missing data, as not all patients completed these measurements at all time points.

$\mathrm{FEV}_{1}$, forced expiratory volume in $1 \mathrm{~s}$; HLC, healthy living course; MBSR, mindfulness-based stress reduction; PEF, peak expiratory flow.

$(p<0.001)$. There were no differences in long-term controller medication use and no change in use over time overall. There was no difference between conditions in days of work or school missed $(p=0.538)$.
Intervention and control sessions attended

Of the nine programme sessions, the mean number attended was 5.64 in the MBSR group and 4.54 in the HLC group $(p=0.047)$.

Table 4 Asthma control, perceived stress, rescue medication and prednisone use: model-derived estimates for baseline, follow-up, and follow-up versus baseline

\begin{tabular}{|c|c|c|c|c|c|c|c|}
\hline & \multicolumn{4}{|l|}{$\%$ (n/total) } & \multicolumn{3}{|c|}{ OR (95\% Cl) follow-up versus baseline } \\
\hline & Baseline & 10 weeks & 6 months & 12 months & 10 weeks & 6 months & 12 months \\
\hline Well controlled asthma & $\mathrm{n}=81$ & $\mathrm{n}=70$ & $\mathrm{n}=75$ & $\mathrm{n}=74$ & & & \\
\hline MBSR & $7.3(3 / 41)$ & $9.1(3 / 33)$ & $21.6(8 / 37)$ & $19.4(7 / 36)$ & $1.3(0.3$ to 5.1$)$ & $3.6(1.1$ to 11.7$)$ & $2.9(0.9$ to 9.7$)$ \\
\hline OR MBSR/OR HLC & & & & & $0.7(0.1$ to 4.4$)$ & $5.4(0.8$ to 38.7$)$ & $2.6(0.4$ to 16.4$)$ \\
\hline $\mathrm{p}$ Value & & & & & 0.689 & 0.093 & 0.301 \\
\hline
\end{tabular}

\begin{tabular}{|c|c|c|c|c|c|c|c|}
\hline & \multicolumn{4}{|l|}{ Mean (SE) } & \multicolumn{3}{|c|}{ Mean change $(95 \% \mathrm{CI})$ from baseline to: } \\
\hline & Baseline & 10 weeks & 6 months & 12 months & 10 weeks & 6 months & 12 months \\
\hline PSS & $\mathrm{n}=81$ & $\mathrm{n}=73$ & $\mathrm{n}=76$ & $\mathrm{n}=77$ & & & \\
\hline MBSR & $17.3(1.1)$ & $13.0(1.0)$ & $12.4(1.0)$ & $13.0(1.0)$ & $-4.3(-6.2$ to -2.3$)$ & $-4.9(-6.6$ to -3.1$)$ & $-4.3(-6.1$ to -2.4$)$ \\
\hline HLC control & $15.8(1.1)$ & $14.3(1.0)$ & $13.1(1.0)$ & $16.0(1.0)$ & $-1.5(-3.5$ to 0.5$)$ & $-2.7(-4.5$ to 0.9$)$ & $0.2(-1.7$ to 2.0$)$ \\
\hline Differential change & & & & & $-2.8(-5.6$ to 0.1$)$ & $-2.1(-4.6$ to 0.4$)$ & $-4.5(-7.1$ to -1.9$)$ \\
\hline \multirow[t]{3}{*}{$\mathrm{p}$ Value } & & & & & 0.055 & 0.097 & 0.001 \\
\hline & \multicolumn{4}{|c|}{ Mean (SE) } & \multicolumn{3}{|c|}{ IRR (95\% CI) follow-up versus baseline } \\
\hline & Baseline & 10 weeks & 6 months & 12 months & 10 weeks & 6 months & 12 months \\
\hline
\end{tabular}

Short-term rescue medication

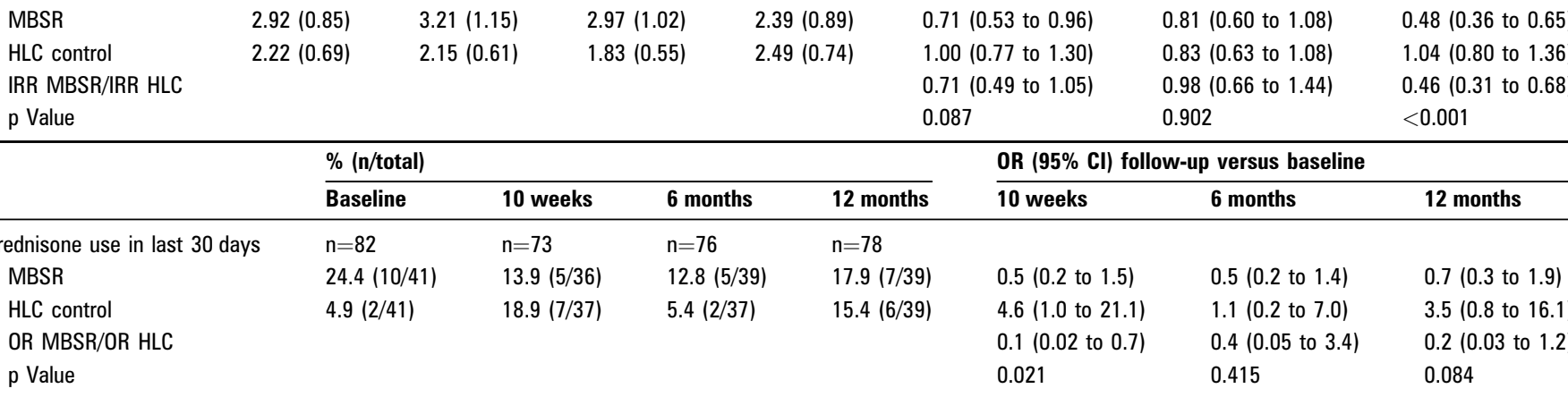

For means, differential change was calculated as the difference in change from baseline for the intervention versus change from baseline for the comparison group. Estimates of ORs comparing follow-up and baseline are from logistic regression models. Estimates of IRRs comparing follow-up and baseline are from a negative binomial regression model. The differing numbers of individuals included in each outcome analysis is due to missing data, as not all patients completed these measurements at all time points.

HLC, healthy living course; IRR, incidence rate ratio; MBSR, mindfulness-based stress reduction; PSS, Perceived Stress Scale. 


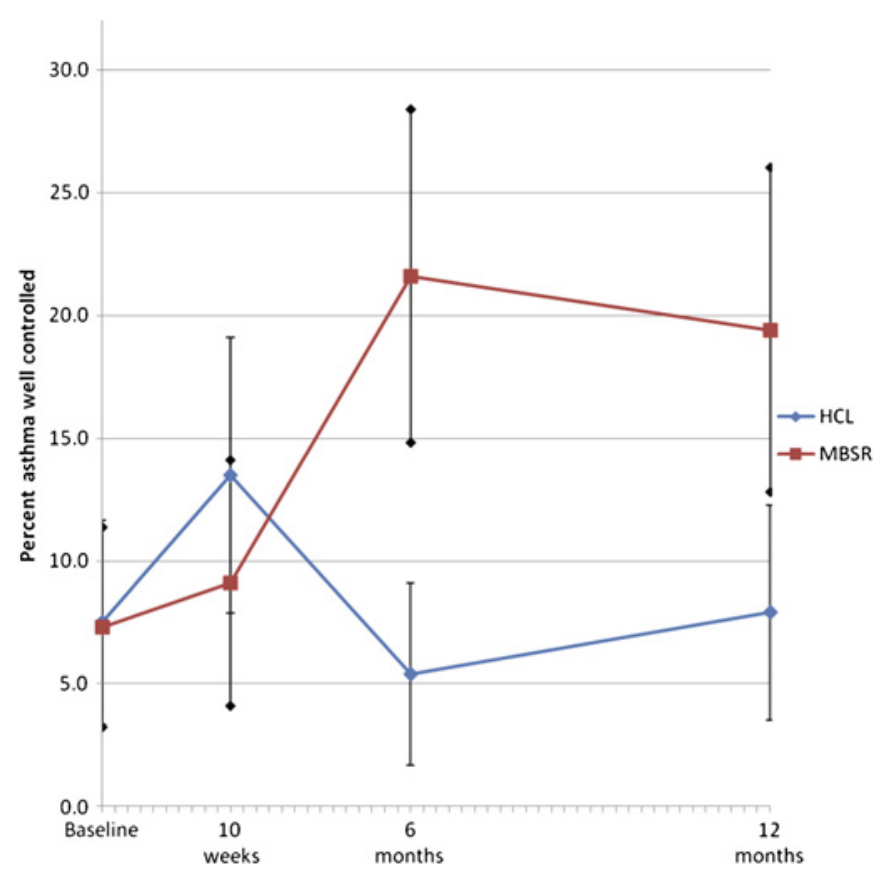

Figure 3 Asthma control. HLC, healthy living course; MBSR, mindfulness-based stress reduction.

\section{DISCUSSION}

Participation in an MBSR programme as an adjunct to asthma treatment resulted in enduring, clinically significant improvements in AQOL in patients with persistent asthma. ${ }^{11}$ For clinical perspective, these are comparable to QOL improvements in trials of widely prescribed asthma medications, including inhaled corticosteroids ${ }^{18}$ and an anti-IgE antibody. ${ }^{19}$ Other complementary programmes have not shown similar definitive improvements in asthma QOL, ${ }^{6}$ suggesting mindfulness training may have uniquely supportive features. The independence of QOL improvements from lung function is consistent with a normative study which also found no correlation between AQOL and lung function. ${ }^{16}$

It was hypothesised that changes in appraisal and reduced reactivity to asthma symptoms from mindfulness training would increase coping resources and affect QOL through decreases in PSS. A number of findings support this model. Baseline PSS (17.3) in the MBSR arm was at the 75th percentile for a US normative sample ${ }^{17}$ and was significantly and substantially reduced to the norm (13.0) at 12 months compared

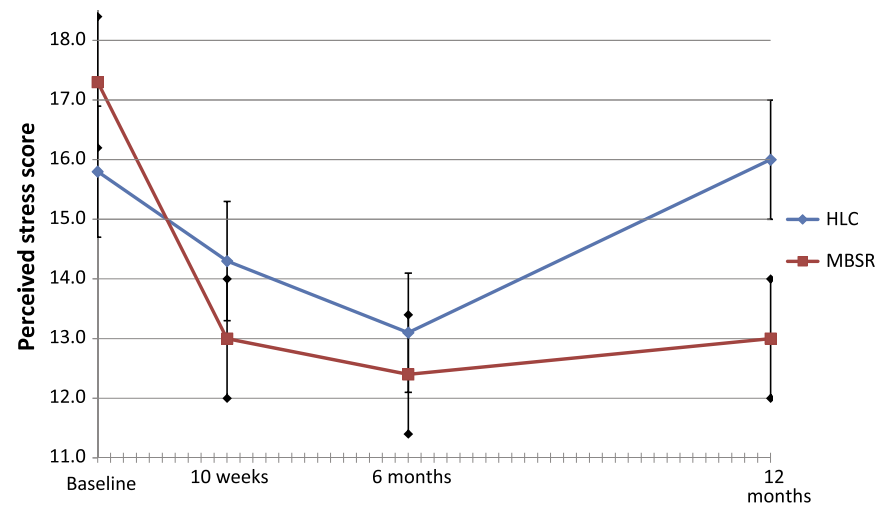

Figure 4 Perceived stress. HLC, healthy living course; MBSR, mindfulness-based stress reduction. with controls, who remained relatively unchanged (from 15.8 to 16.0). Together with the finding that the largest effect in AOOL was in emotional function, this suggests coping resources increased in patients in the MBSR group. The finding of reduced stress has important clinical implications because higher stress levels are associated with increased asthma morbidity, poorer QOL and suboptimal disease management. ${ }^{2}$

The lack of accompanying improvements in lung function has implications for debate on the causal relation between stress and asthma. Laboratory-induced stress increases airway resistance in patients with asthma, ${ }^{20}$ but longer-term life stress is not associated with lung function. ${ }^{21}$ Our findings support the view that life stress, while affecting asthma QOL, may not contribute to impairment in lung function.

An interesting and encouraging finding is the pattern of change between treatment conditions over 12 months. While both conditions tended towards improvement in QOL, stress and asthma control in the first 6 months, the smaller gains achieved by controls largely deteriorated to baseline levels at 12 months. The MBSR programme maintained or improved their gains without further intervention. The HLC programme may have produced an initial placebo effect, a suggestion supported by a recent trial demonstrating some placebo effect of bronchial thermoplasty on QOL in patients with asthma, ${ }^{22}$ and a cognitive behavioural intervention trial for patients with asthma and panic disorder that found AOOL gains during an 8 -week intervention were not sustained at 6 months. ${ }^{23}$ To our knowledge this is the first randomised trial using an active control that has followed MBSR participants for 12 months. It may be that more accurate discrimination between thoughts, sensations and feelings, and the reduced urgency and distress resulting from non-reactive awareness associated with mindfulness training is a lasting perceptual change. ${ }^{24}$

The study addresses criticism that studies of mindfulness training ${ }^{25}$ and complementary programmes used by patients with asthma ${ }^{6}$ lack credible active controls, include only subjective endpoints, inadequately define participant characteristics and treatment methods, and fail to control for the supportive interaction with a practitioner, the most potent component of non-specific effects in medical encounters. ${ }^{26}$ While the nonspecific effects of the MBSR and control interventions could differ, the finding that increased mindfulness from MBSR programmes mediates reductions in $\mathrm{PSS}^{27}$ adds support to mindfulness training as the active ingredient. Additionally, by enrolling participants in a widely available programme rather than an experimental programme tailored to patients with asthma, the study addresses the more general criticism that behavioural trials create intervention conditions that are not generalisable, or translatable into the 'real world'. It is reasonable to expect similar findings in the approximately $200 \mathrm{MBSR}$ programmes available at US and European healthcare facilities, many of which are covered by third party payers, making this a realistic adjunctive asthma treatment.

There were a number of study limitations. The modest sample size of this pilot study limited power to detect differences in a number of variables, including asthma control, and the ability to detect whether the intervention effect was specific to patients with asthma of a particular severity and whether it was related to changes in concomitant medications. Also, a substantial number of results have been calculated in a fairly restricted dataset; some results may be due to chance, especially when $p$ values are close to 0.05 . Generalisability of the findings is limited by a number of factors. First, patients were recruited from a pulmonary medicine and primary care population in an 
academic medical centre and may not be representative of the general asthma patient population. Second, refusal rate was high among eligible patients (71\%), raising concerns about the selfselected nature of the sample. However, while our rate is higher than some other randomised controlled trials of behavioural interventions (approximately $37 \%)^{28} 29$ it is similar to the $75 \%$ who declined a randomised controlled trial of a behavioural programme for asthma. ${ }^{30}$ The challenge of recruiting participants to clinical trials is widespread, with many factors involved in refusal that may have little to do with the generalisability of the intervention. ${ }^{31} 32$ The most frequent reason for declining to participate in the present study was being too busy to participate in a clinical trial (23\%), suggesting the trial requirements were a barrier, rather than a rejection of the intervention itself. Third, the majority of patients in the trial were white, nonLatino and of higher socioeconomic status. Lastly, attendance at five of the nine MBSR sessions suggests its traditional delivery may not be suitable for people with a chronic illness, and that a shorter programme may be equally effective. ${ }^{33}$

Acknowledgements We gratefully acknowledge the technicians in the UMMHC Pulmonary Diagnostic Laboratory for conducting the spirometry, and Dr Sarah ReiffHekking for conducting the Healthy Living Course (compensated financially for their work on this study).

Contributors All authors participated in study conception and design, analysis and interpretation of data, and drafting and revising of the manuscript and all authors approved the manuscript as submitted.

Funding Study supported by grant R21 AT002938 (awarded to Drs Pbert and Carmody) from the NIH National Center for Complementary and Alternative Medicine. The sponsor provided financial support only and had no role in the design, conduct, data collection, management, analysis, and interpretation of the study; or in the preparation, review, or approval of the manuscript.

\section{Competing interests None.}

Ethics approval Ethics approval was provided by the Institutional Review Board.

Provenance and peer review Not commissioned; externally peer reviewed.

Data sharing statement Drs Pbert and Carmody had full access to all of the data and take responsibility for the integrity and the accuracy of the data and analysis.

\section{REFERENCES}

1. Lemmens KM, Nieboer AP, Huijsman R. A systematic review of integrated use of disease-management interventions in asthma and COPD. Respir Med 2009;103:670-91.

2. Wisnivesky JP, Lorenzo J, Feldman JM, et al. The relationship between perceived stress and morbidity among adult inner-city asthmatics. J Asthma 2010;47:100-4.

3. Janssens T, Verleden G, De Peuter S, et al. Inaccurate perception of asthma symptoms: a cognitive-affective framework and implications for asthma treatment. Clin Psychol Rev 2009;29:317-27.

4. Rietveld S. Symptom perception in asthma: a multidisciplinary review. J Asthma 1998:35:137-46.

5. Yorke J, Fleming SL, Shuldham C. Psychological interventions for adults with asthma: a systematic review. Respir Med 2007;101:1-14.

6. Markham AW, Wilkinson JM. Complementary and alternative medicines (CAM) in the management of asthma: an examination of the evidence. J Asthma 2004:41:131-9.
7. Shigaki CL, Glass B, Schopp LH. Mindfulness-based stress reduction in medical settings. J Clin Psychl Med Settings 2006;13:209-16.

8. National Heart Lung and Blood Institute (NHLBI). Expert Panel Report 3. Guidelines for the Diagnosis and Management of Asthma. NIH Publication No. 074051. US Department of Health and Human Services, 2007. http://www.nhibi.nih. gov/guidelines/asthma/asthgdin.htm (accessed 11 Apr 2012).

9. Kabat-Zinn J. Full Catastrophe Living: Using the Wisdom of Your Body and Mind to Face Stress, Pain and IIIness. New York: Delacorte, 1990.

10. Juniper EF, Buist AS, Cox FM, et al. Validation of a standardized version of the asthma quality of life Questionnaire. Chest 1999;115:1265-70.

11. Juniper EF, Guyatt GH, Willan A, et al. Determining a minimal important change in a disease-specific quality of life questionnaire. J Clin Epidemiol 1994;47:81-7.

12. Anon. Standardization of spirometry, 1994 update. American Thoracic Society. Am J Respir Crit Care Med 1995;152:1107-36.

13. Reddel HK, Taylor DR, Bateman ED, et al. An official American Thoracic Society/ European Respiratory Society statement: asthma control and exacerbations: standardizing endpoints for clinical asthma trials and clinical practice. Am J Respir Crit Care Med 2009;180:59-99.

14. Cohen S, Kamarck T, Mermelstein R. A global measure of perceived stress. J Health Soc Behav 1983:24:385-96.

15. Boushey HA, Sorkness CA, King TS, et al. Daily versus as-needed corticosteroids for mild persistent asthma. N Engl J Med 2005;352:1519-28.

16. Leidy NK, Coughlin C. Psychometric performance of the Asthma Quality of Life Questionnaire in a US sample. Qual Life Res 1998;7:127-34.

17. Cohen S, Williamson C. Perceived stress in a probability sample of the United States. In: Spacapan S, Oskamp S, eds. The Social Psychology of Health. Newbury Park, CA: Sage, 1998:31-67.

18. Boulet LP, Bateman ED, Voves $\mathrm{R}$, et al. A randomized study comparing ciclesonide and fluticasone propionate in patients with moderate persistent asthma. Respir Med 2007; 101:1677-86

19. Holgate ST, Chuchalin AG, Hebert J, et al. Efficacy and safety of a recombinant antiimmunoglobulin E antibody (omalizumab) in severe allergic asthma. Clin Exp Allergy 2004;34:632-8.

20. Ritz T, Kullowatz A. Effects of emotion and stress on lung function in health and asthma. Curr Respir Med Rev 2005;1:209-18.

21. Kullowatz A, Rosenfield D, Dahme B, et al. Stress effects on lung function in asthma are mediated by changes in airway inflammation. Psychosom Med 2008;70:468-75.

22. Castro $\mathbf{M}$, Rubin AS, Laviolette $\mathbf{M}$, et al. Effectiveness and safety of bronchial thermoplasty in the treatment of severe asthma: a multicenter, randomized, doubleblind, sham-controlled clinical trial. Am J Respir Crit Care Med 2010;181:116-24.

23. Ross CJ, Davis TM, MacDonald GF. Cognitive-behavioral treatment combined with asthma education for adults with asthma and coexisting panic disorder. Clin Nurs Res 2005; 14:131-57

24. Carmody J. Evolving conceptions of mindfulness in clinical settings. $J$ Cogn Psychotherapy 2009:23:270-80.

25. Ludwig DS, Kabat-Zinn J. Mindfulness in medicine. JAMA 2008;300:1350-2.

26. Kaptchuk TJ, Kelley JM, Conboy LA, et al. Components of placebo effect: randomised controlled trial in patients with irritable bowel syndrome. $B M$ 2008;336:999-1003.

27. Carmody J, Baer RA, L B Lykins E, et al. An empirical study of the mechanisms of mindfulness in a mindfulness-based stress reduction program. J Clin Psychol 2009;65:613-26.

28. Karlson CW, Rapoff MA. Attrition in randomized controlled trials for pediatric chronic conditions. J Pediatr Psychol 2009;34:782.

29. Sidani S, Bootzin RR, Moritz P, et al. Patterns of enrollment in randomized and preference trials of behavioral treatments for insomnia. J Methods Meas Soc Sci 2011;1:15-30.

30. Put C, van den Bergh 0, Lemaigre $\mathrm{V}$, et al. Evaluation of an individualised asthma programme directed at behavioural change. Eur Respir J 2003;21:109-15.

31. Ellis PM. Attitudes towards and participation in randomised clinical trials in oncology: a review of the literature. Ann Oncol 2000:11:939-45.

32. Gul RB, Ali PA. Clinical trials: the challenge of recruitment and retention of participants. J Clin Nurs 2010;19:227-33

33. Carmody J, Baer RA. How long does a mindfulness-based stress reduction program need to be? A review of class contact hours and effect sizes for psychological distress. J Clin Psychol 2009;65:627-38. 